\title{
Odor Discrimination by Mitral Cells in Rat Olfactory Bulb Using Microwire Array Recordinge
}

\author{
Q. Dong, J. Zhou, L.J. Zhuang, D. Wang, P. Wang* \\ Biosensor National Special Laboratory, Key Laboratory for Biomedical Engineering of Education \\ Ministry, Department of Biomedical Engineering, Zhejiang University, Hangzhou, China \\ ${ }^{*}$ Corresponding author: cnpwang@zju.edu.cn
}

\begin{abstract}
Response features of mitral cells in the olfactory bulb were examined using principal component analysis to determine whether they contain information about odorant stimuli. Using microwire electrode array to record from the olfactory bulb in freely breathing anesthetized rats, we recorded responses of different mitral cells to saturated vapor of anisole $(10 \mathrm{mM})$, carvone $(10 \mathrm{mM})$, isobutanol $(10 \mathrm{mM})$, citral $(10 \mathrm{mM})$ and isoamyl actate $(10 \mathrm{mM})$. The responses of single mitral cells to the same odorant varied over time. The response profiles showed similarity during certain amount of period, which indicated that the response was not only depended on odor itself but also associated with context. Furthermore, the responses of single mitral cell to different odorants were observed with difference in firing rate. In order to recognize different odorant stimuli, we apply four cells as a sensing group for classification using principal component analysis. Features of each cell's response describing both temporal and frequency characteristics were selected. The results showed that five different single molecular odorants can be distinguished from each other. These data suggest that action potentials of mitral cells may play a role in odor coding.
\end{abstract}

Key words: mitral cell, odor discrimination, context-based response and anesthetized rat

\section{Introduction}

Smell carries important cues about food, natural enemy, and social information through large numbers of volatile odorants, which the olfactory system needs accurately identify. Odor information is ultimately encoded in the mammalian olfactory bulb (OB) by action potentials of mitral/tuft cells, which form its output to cortex. Odor-specific distributed representations can be observed via imaging studies of OB glomeruli [1-2], or be detected via electrophysiological studies by microelectrodes in the mammalian OB [3-7]. Multielectrode array increases the probability of successful recording. Meanwhile, simultaneous or nearsimultaneous electrophysiological recordings of multiple neurons may contain additional information about a stimulus that is available only at the ensemble level [8].

Most researches about how OB works have been based on anesthetized preparations. Initial studies only focused on firing rate, interspike interval and amplitude of odorant responses. Further studies are more based on odor coding and olfactory map [9]. Study on recordings from the same mitral cell in anesthetized and awake states has demonstrated that odor response in the anesthetized state is stronger than in the awake state, the amplitude of response is larger and the firing rate is higher [10].

In this report we used a multielectrode array implanted in the OB of rat to investigate how 1) single mitral cell respond to repeated stimulations by the same odorant; 2) single mitral cell respond to different odorants; 3 ) different mitral cells respond to the same odorant; and 4) discrimination of odorants with different functional groups by context-based mitral cells.

\section{Materials and methods \\ Male Sprague-Dawley rats (180-280 g) were anesthetized with an intraperitioneal injection of chloral hydrate $(0.4 \mathrm{ml} / 100 \mathrm{~g})$ and were held in a standard stereotaxic apparatus. A craniotomy was performed to gain access to the dorsal OB. The designed 8-channal microwire electrode array was implanted to a depth of nearly 300 - $400 \mu \mathrm{m}$ in one hemisphere of the dorsal OB which corresponds to the average depth of the mitral cell layer (Fig. 1). The criteria of mitral}


cell search were both from the stereotaxic coordinates of the dorsal $\mathrm{OB}$ and signal amplitude in which the mitral cell was larger than other cells' in the bulb [11]. The electrode array insertion tracks were checked with postmortem histological staining. After insertion, blood in the implant site was washed away with saline and the surface of the $O B$ was rinsed with saline to prevent dehydration, the array was then connected to an 8-channel amplifier and data acquisition system (USB-ME16-FAISystem, MultiChannel Systems MCS $\mathrm{GmbH}$, Inc). Array was advanced using a hydraulic pressure-microelectrode propeller so that the depth of the implantation could be successfully fine-tuned to achieve simultaneously recording of single- and multi-unit activities. All animal care and experimental procedures were in strict accordance with a protocol approved by the Zhejiang University Animal Care and Use Committee.

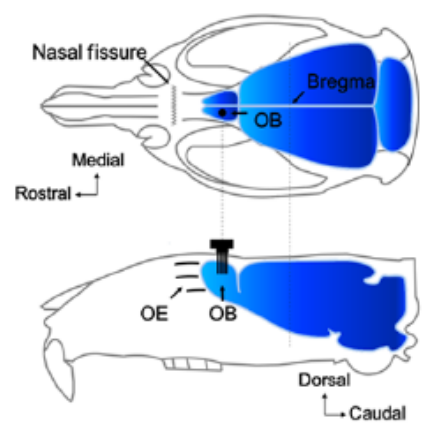

Fig. 1. The anatomical position of electrode implantation

We used small molecular odorants including anisole, citral, carvone, isobutanol, and isoamyl acetate as stimuli, which were stored in liquid phase in glass vials with concentration of 1 $\mathrm{mol} / \mathrm{L}$. The odorant delivered to freely breathing rat was the saturated vapor from the head space of vials. One stimulation session was to puff one of five odorants to rat nose using $2 \mathrm{ml}$ syringe for $1 \mathrm{~s}$ and interleaved clean air for $60 \mathrm{~s}$, then repeated the procedure with another odorant until all five odorants had been applied. The response evoked by the odorant delivery without airflow before stimulation can't be attributed to the odorant only.

Signals from individual electrode were amplified by 1000 times, digitized at $20 \mathrm{KHz}$, filtered between 1 and $5 \mathrm{KHz}$, and simultaneously recorded on a laptop for off-line analysis. The animal's breathing was simultaneously monitored through a sensitive pressure sensor near the rat's lung throughout the course of the experiment (2-4 h).

\section{Results and discussion}

Mitral cell activities were recorded using an eight channel electrode array. In 30 stimulation sessions we obtained 16 unambiguously discriminated individual cells from three animals. The firing rates of the cells ranged from 1 to 10 $\mathrm{Hz}$.

Most of the mitral cells responded to the odorants weakly or intermittently modulated by respiration. The response of mitral cell to same odorant was not firing in uniform rate, although the action potential waveform shares the common shape and size. Figure 2 shows a single mitral cell responds to anisole stimulation. Every trial was recorded in ten respiration cycles. Trial-trial interval was 6 minutes. The mean firing rates were from 1.6 to $3.7 \mathrm{~Hz}$. Compared the rate histogram of each trial, this neuron showed different bursting behavior. In some sessions the firing was very sparse, but in other sessions it was more actively. The adjacent trials represented like trial 1 and trial 2 . Responses of single mitral cell to the rest of all the five odors were also examined. The firing rate varied over time. The firing profiles of the single cell indicated that the response pattern to the same odor was not only depended on the odor itself, but also context associated.

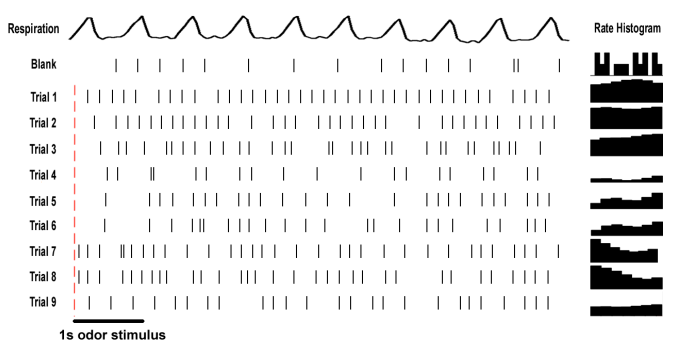

Fig. 2. Single mitral cell responded to repeated anisole stimulation.

Then, we detected the response of a single mitral cell to different odorants. Figure 3 shows one mitral cell responds to clean air (blank) and five odorants. The firing rate of the mitral cell to clean air was $1.6 \pm 0.3 \mathrm{~Hz}($ Mean $\pm S D, n=8)$. This mitral cell behaved more actively to isoamyl acetate, with a firing rate at $3.5 \pm 0.2 \mathrm{~Hz}$. The responses to anisole, carvone and citral were moderate, the mean firing rate was around $2 \mathrm{~Hz}$. The response to isobutanol was similar to blank, which indicates that this neuron was not activated by isobutanol. As previous study demonstrated that each olfactory receptor neuron expresses only one gene of the receptor family [12], and axons of ORNs that express the same receptor converge into two glomeruli within the main $\mathrm{OB}$, one at the lateral and the other, the medial side. Odorants with similar 
chemical structures activate glomeruli within certain regions of the $\mathrm{OB}$. Thus, single mitral cell might be more sensitive to one kind of odorant, but not suitable for odor classification.

Single mitral cell is not widely responsive to all odorants. Through visualized olfactory sensory map, we can clearly see the topographical projection from ORN to glomeruli [13]. We believe that more mitral cells should join in for odor recognition. The mitral cells exhibit different responses towards one odorant. Some cells respond specific to particular stimulus, some cells do not respond to that stimulus, and some cells are even inhibited by that stimulus. The cell group activity might have certain pattern for different odor stimulus. Because the response of mitral cell to the same odorant varied over time, continuous sessions with similar firing histogram were selected for odor discrimination analysis. The raw data was firstly processed by four-order butterworth high pass filter. The cutoff frequency was set to $60 \mathrm{~Hz}$ for filtering low frequency noise, which stabilized the signal baseline. Then, we measured the standard deviation of the response amplitude during the first 200 samples of spike-free data to quantify the level of baseline noise. The threshold was chosen 3 times SD of the baseline noise empirically (shown in Fig. 4a).

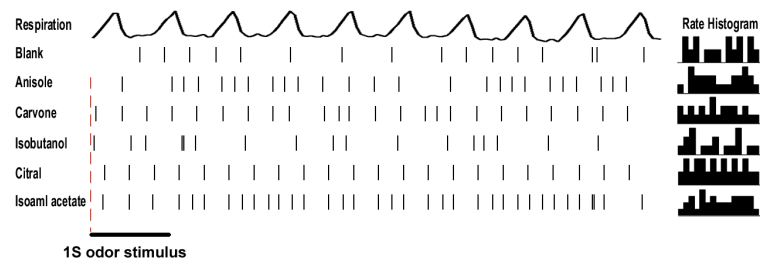

Fig. 3. Single mitral cell responded to five odorants stimulation.

In order to quantitatively compare responses to different odorants, we chose four cells as one cell group and characterized each mitral cell response by three features including firing rate, mean inter-spike interval (ISI) and wavelength (shown in Fig. 4b). Firing rate was calculated in first ten respiration cycle when the odor stimulus begins, which included frequency information. Mean ISI provides temporal information for each response. Wavelength is the time interval chosen from peak to valley, which provides different cell waveforms information related to odor stimuli.

The data matrix for principal component analysis (PCA) was from three continuous stimulation sessions. The responses were from mitral cell group which includes four mitral cells. Three features of each mitral cell were selected. The size of the data matrix is $15 \times 12$. After data normalization, covariance calculation, and eigenvalue sorting, two eigenvectors were chosen for PCA. First principal component's contribution rate is $70.4 \%$ and second principal component's contribution rate is $20.3 \%$. Total contribution rate is larger than $90 \%$ which is significant for analysis. The context based odor discrimination can be achieved by principal component analysis method (Shown in Fig. 4c).

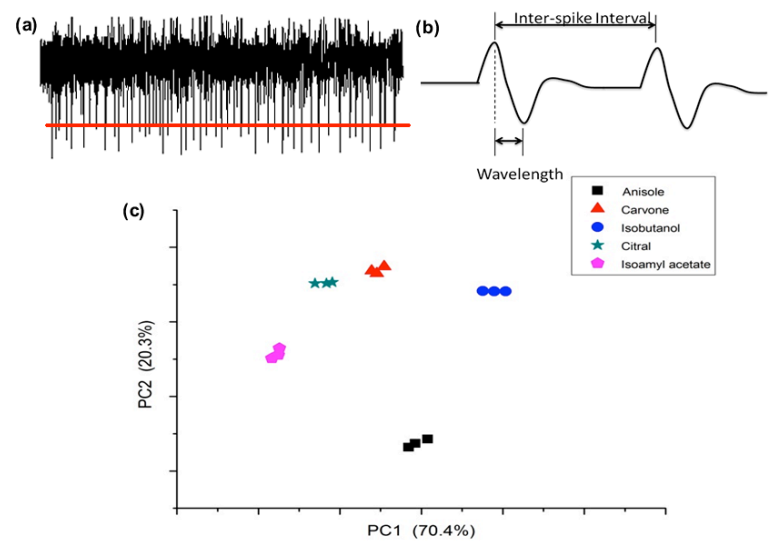

Fig. 4. Results of principal component analysis. (a) The threshold was chosen 3 times SD of the baseline noise. (b) Three feature selection including firing rate, mean inter-spike interval and wavelength. (c) Principal component analysis for five odorants stimulation.

\section{Conclusion}

In this paper we have presented data showing that information regarding odor stimulus is present in the firing patterns of mitral cell group. More specifically, we have shown that principal component analysis can discriminate responses to anisole, carvone, isobutanol, citral and isoamyle actate. In addition, response of single mitral cell to one odorant varies with environment. Interestingly, responses to different odorants have specific features. The mean firing rate, inter-spike interval and waveform length occurred differently. These results suggest that cell group can provide more stable and informative response towards different odorant stimuli.

This study shows that odor stimuli can be discriminated solely based on features relating to the firing rate of the response and patterns of action potentials of the mitral cell group. Due to the dimension of electrode array, the number of mitral cells in cell group was limited. It is assumed that by "listening" to more mitral cells can distinguish more precisely different odor stimuli. Besides, extracellular recordings are invariably in favor of neurons that fire and respond to the stimulus. Thus, we may miss a number of mitral cells that remained silent to odors, and therefore the stated fraction of 
responsive mitral cells does not well represent the cell group response. Further, larger electrode array will be applied to obtain more mitral cell responses.

\section{Acknowledgements}

This research is supported by the National Natural Science Foundation of China (Grant 60725102).

\section{References}

[1] B.D. Rubin and L. C. Katz, "Optical Imaging of Odorant Representations in the Mammalian Olfactory Bulb", Neuron 23: 499-511(1999).

[2] M. Leon and B. A. Johnson, "Olfactory coding in the mammalian olfactory bulb", Brain Res. Rev. 42: 23-32(2003).

[3] M.A. Chaput, A. Holley, "Responses of olfactory bulb neurons to repeated odor stimulations in awake freely-breathing rabbits", Physiol. Behav. 34: 249-258(1985).

[4] M.J. Lehmkuhle, R.A. Normann, E.M. Maynard, "High-resolution analysis of the spatio-temporal activity patterns in rat olfactory bulb evoked by enantiomer odors", Chem. Senses. 28: 499508(2003).

[5] 5. F. Motokizawa, "Odor representation and discrimination in mitral/tufted cells of the rat olfactory bulb", Exp. Brain. Res. 112: 2434(1996).
[6] J. Leveteau, P. MacLeod, "Olfactory discrimination in the rabbit olfactory glomerulus", Science 153: 175-176(1966).

[7] U.S. Bhalla, J.M. Bower, "Multiday recordings from olfactory bulb neurons in awake freely moving rats: spatially and temporally organized variability in odorant response properties", J. Comput. Neurosci. 4: 221-256(1997)

[8] N. Buonviso, M.A. Chaput, F. Berthommier, "Temporal pattern analyses in pairs of neighboring mitral cells", J. Neurophysiol. 68: 417-424(1992).

[9] M. Chalansonnet, M.A. Chaput, "Olfactory bulb output cell temporal response patterns to increasing odor concentrations in freely breathing rats", Chem. Senses. 23: 1-9(1998).

[10] M.J. Lehmkuhle, R.A. Normann, E.M. Maynard, "Trial-by-trial discriminationof three enantiomer pairs by neural ensembles in mammalian olfactory bulb” , J. Neurophysiol. 95:1369 1379(2006).

[11] I.G. Davison, L.C. Katz Sparse, "Selective odor coding by mitral/tufted neurons in the main olfactory bulb", J. Neurosci. 27:2091-2101(2007).

[12] 10. D. Rinberg, A. Gelperin, "Olfactory neuronal dynamics in behaving animals", Seminars in Cell \& Developmental Biology 17:454-461(2006).

[13] J. Cang, J.S. Isaacson, "In vivo whole-cell recording of odor-evoked synaptic transmission in the rat olfactory bulb", J. Neurosci. 23:41084116(2003). 